\title{
MAGNETIC DRIVE SYSTEM FOR A NEW CENTRIFUGAL ROTARY BLOOD PUMP
}

Authors: Andrew Hilton ${ }^{1}$, Geoff Tansley ${ }^{2}$

${ }^{1}$ The University of Nottingham, School of Mechanical, Materials and Manufacturing Engineering

${ }^{2}$ Aston University, School of Engineering and Applied Science

Address correspondence to:

\section{Mr A Hilton}

Room: 301 Wolfson Building

The University of Nottingham

University Park

Nottingham

NG7 2RD

Telephone: +44(0)115846 6407

Email: eaxah2@nottingham.ac.uk

Magnetic drive for CRBP 


\begin{abstract}
Introduction: The purpose of this investigation was to design a novel magnetic drive and bearing system for a new Centrifugal Rotary Blood Pump (CRBP).

Methods: The drive system consists of two components: permanent magnets within the impeller of the CRBP and the driving electromagnets. Orientation of the magnets varies from axial through to $60^{\circ}$ included out-lean (conical configuration). Permanent magnets replace the electromagnet drive to allow easier characterization. The performance characteristics tested were the axial force of attraction between the stator and rotor at angles of rotational alignment, $\varnothing$, and the corresponding torque at those angles. The drive components were tested for various magnetic cone angles, $\theta$. The test was repeated for three backing conditions: non-backed, steel-cupped and steel plate back-iron; performed on an Instron tensile testing machine. Experimental results were expanded upon through FEA / BEA analysis.

Results: The force / torque characteristics were maximal for a 12 magnet configuration at $0^{\circ}$ cone angle with steel back-iron $($ axial force $=60 \mathrm{~N}$, torque $=0.375 \mathrm{Nm}$ ). FEA $/$ BEA analysis showed how introducing a cone angle increases the radial restoring force three-fold, whilst not compromising axial bearing force. Conclusions: Magnets in the drive system may be orientated not only to provide adequate coupling to drive the CRBP, but to provide significant axial and radial bearing forces. Although the 12 magnet $0^{\circ}(\theta)$ configuration yielded the greatest force / torque characteristic, this was seen as potentially unattractive as this magnetic cone angle yielded poor radial restoring force characteristics.
\end{abstract}

Key Words: magnetics, prostheses and orthotics, rotary blood pump 


\section{Introduction}

The purpose of this investigation was to design a magnetic drive system for a centrifugal rotary blood pump (CRBP) that will be used as a Left Ventricular Assist Device (LVAD). It was proposed in the design to incorporate electromagnets into the pump casing that not only serve as the drive system to the CRBP, but as part of the hybrid non-contact bearing. A convenient starting point though was a permanent magnetic coupling; once the drive and bearing systems have been proven to work satisfactorily, translation of the permanent coupling to an electromagnetic one will be undertaken. Magnetic suspension with permanent magnetic bearings offers a number of advantages, namely zero power consumption, extreme reliability and longevity, and substantially higher operating speeds relative to conventional mechanical bearings. In magnetic suspension, permanent magnets are used to provide passive levitation and or positioning of a structure ${ }^{1}$.

It is well documented that a passive magnetic field alone cannot provide stable support of a structure. This is a consequence of Earnshaw's (1842) theorem, which states: "A collection of point charges cannot be maintained in a stable stationary equilibrium configuration solely by the electrostatic interaction of the charges". As a consequence, passive magnetic bearings cannot be used exclusively; it can be shown that "never will a radial bearing be axially stable nor will a thrust bearing be radially stable" ${ }^{2}$. They must therefore be used in conjunction with a mechanical system or active magnetic bearings in order to achieve complete suspension. A passive magnetic bearing will be either a radial bearing if it controls a rotation axis, or an axial one, when it maintains the position of the rotor against displacement along the axis of rotation ${ }^{3}$. The proposed design here incorporates both radial and axial bearing forces.

In order to evaluate the forces between two magnets some simplifying assumptions are made. The magnetic material is assumed to be very strong (e.g. rare earth magnets such as $\mathrm{NeFeB}$ magnets are modelled). For rotating applications, the separation between the magnets is small in comparison to the radius of the bearing. The length of the magnet perpendicular to the cross section is large compared to the gap size, and thus flux lines are organised in cross sectional planes. This yields a two-dimensional problem. The equations and figures below outline the magnetic arrangement and establish the key forces, dimensions and 
angles. It must be noted at this point that we are considering multi-pole ring magnets with an alternating flux density vector through the thickness of the ring.

$$
\begin{aligned}
& \text { Axial force }=F_{z} \approx \frac{-B_{r 1} B_{r 2}}{2 \pi \mu} \frac{2}{R^{3}} A_{1} A_{2} \sin \\
& \text { Radial force }=F_{x} \approx \frac{-B_{r 1} B_{r 2}}{2 \pi \mu} \frac{2}{R^{3}} A_{1} A_{2} \cos { }_{-}
\end{aligned}
$$

Where: $F_{x}, F_{y}=$ Force in $x$ and $y$ directions respectively $(\mathrm{N})$

$$
B_{r}=\text { Magnetic flux density }(\mathrm{T})
$$

$R=$ Separation between magnets $(\mathrm{m})$

$A=$ Cross-sectional area of the magnets $\left(\mathrm{m}^{2}\right)$

$\alpha=$ Orientation angle between magnet flux density vectors (degrees)

$K_{x, z}=$ Magnetic stiffness in the $x$ and $z$ direction

$\mu=$ Magnetic permeability (equals the product of the permeability of free space, $\mu_{0}$, and he relative permeability of the material in the separation gap, $\mu_{r} . \quad \mu=\mu_{0} \mu_{r}$

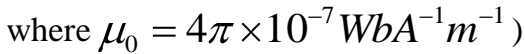

Subscripts ${ }_{1}$ and ${ }_{2}$ denote magnets 1 and 2 .

The force between facing magnets is always an attractive force, regardless of the magnet arrangement ${ }^{3}$; this is a key fact in incorporating the drive system as part of the bearing.

The magnetic bearing stiffness can be examined using Equation (3). (C = Integration constant).

$$
K_{z} \approx \frac{B_{r 1} B_{r 2}}{2 \pi \mu} \frac{6}{R^{4}} A_{1} A_{2} \cos (2 \alpha)=C \cos (2 \alpha)
$$

Examining Equation (3), solving for $C$ : 
$\alpha$-type: $C=K_{x}=-\frac{K_{z}}{2} \quad \beta$-type: $C=K_{x}=K_{z}=0 \quad \gamma$-type: $C=-K_{x}=\frac{K_{z}}{2}$.

The $\alpha$-type leads to highly unstable radial stiffness, but the axial force is null for the middle position. The $\beta$-type does not produce any significant stiffness, (The $\beta$-type is therefore advantageous in applications where the strains on the bearings have to be minimised.) The $\gamma$-type leads to a high axial force and contributes to maintaining the rotation in a centred position. Therefore for an application of a magnetic coupling as suggested by the design of this CRBP, it is desirable to have a $\gamma$-type coupling ${ }^{3}$. Bearing types are displayed in Error! Reference source not found..

Discussion so far has centred on the arrangement of ring magnets with alternating magnetic flux densities to produce a coupling with the desired bearing potential. If we now consider replacing the ring magnets with a number of identical small disc magnets, the same bearing configuration may be achieved by inverting every other disc magnet, thus forming an alternating magnetic field similar to that produced by the ring magnets (Figure 2).

\section{Physical Testing - Attractive force between the two parts of the drive system}

The attractive force between the two components of the coupling was measured. A number of samples (magnet holders) were drawn up using Pro/ENGINEER and subsequently 3-D printed using a rapid prototype machine. These samples show some potential magnetic arrangements which could be used within the CRBP, including a range of angled, conical surfaces $(\theta)$ from $0^{\circ}$ to $30^{\circ}$ in $10^{\circ}$ increments (Figure 3 ). The upper component represents the impeller whilst the lower represents the pump casing. The upper and lower samples can accept between 0 and 8 individual magnets each; a 12 magnet configuration sees 6 magnets housed in the lower sample and 6 in the upper. Neodymium Iron Boron magnets were used of dimensions: $8 \mathrm{~mm} \varnothing$ by $5 \mathrm{~mm}$ - grade $\mathrm{N} 42$. The magnets sit on a pitch circle diameter of $30 \mathrm{~mm}$. The samples were designed to clamp into an Instron tensile testing machine Testing is illustrated in Figure 4. 
The Instron is capable of measuring force as a function of displacement. The $50 \mathrm{~N}$ load cell used is accurate to 0.2 Newton. The test was started with the two components in contact, with the magnets fully aligned (in $\emptyset)$, as shown in Figure 4 the upper piece was drawn vertically away from the lower. The test was repeated for 8 and 12 magnet configurations and under 3 different backing conditions: un-backed, cupped (the magnets are placed into individual steel cups) and plain-backed (i.e. steel washer). The measured force is plotted against separation and is shown in the pull-gap curves of Figure. It is important to note that the smallest separation possible is $2 \mathrm{~mm}$ due to the design of the samples - this distance is due to the material preventing the magnets from touching. Table 1 outlines the magnet arrangements tested.

\section{Results and Discussion of Physical Testing}

Figure 5 displays the results of the physical testing.

The smaller the separation gap, the greater the force of attraction. Higher numbers of magnets gave a greater force of attraction: 8 magnets at the same separation show gave $25 \mathrm{~N}$ (Figure 5a), and 12 magnets gave a total attractive force of $38 \mathrm{~N}$ (Figure 5b). Angling the surface of the components, and thus the angle of the magnetic flux vector, affects the axial attraction force. This is due to the cosine component of the vertical force decreasing as the cone angle increases; the greater the cone angle, the lower the force of attraction. There is no discernable difference between arrangements at $10^{\circ}$ and those at $20^{\circ}$. Steel cupping of the magnets decreases the attractive force by approximately $25 \%$ (Figure $5 \mathrm{~b}$ ). Plain-backing the magnets increases the attraction by approximately $160 \%$ (Figure $5 b$ ). 


\section{Computational Modelling}

The results of the physical testing are valid only for magnet configurations that are completely aligned around the z-axis (in $\varnothing$ ). In reality, when the drive system is operational, there will be frictional forces retarding the rotation of the impeller. These frictional forces will cause the two components of the drive system to become misaligned - the rotor will lag the magnetic field produced by the stator. It was therefore necessary to measure how the force of attraction between the parts varies at angles of misalignment, $\varnothing$; this was modelled using FEM / BEM analysis. Amperes (Integrated Engineering Software, Winnipeg, Canada) is a CAE software package designed to perform full 3D simulations of magnetic physical systems. It provides users with a wide variety of analysis options, including the ability to create contour plots and graphs of field quantities. To perform a simulation in Amperes a geometric model of the physical system was constructed. This was done using the built-in geometric modeller although it is possible to import files directly for CAD packages. Once the geometric model was built, physical properties such as material and magnet flux density vector orientation were assigned. After the physical properties have been assigned, the model was discretized and the solution was calculated by the field solver. (The user can either manually discretize the model or having the self-adaptive solver perform the discretization. This investigation uses the latter.) This software enabled quick and efficient modelling of the samples that had been manufactured, allowing further analysis that would be difficult to perform experimentally. FEA / BEM analysis yielded results for the axial force and the torque between the components at angles of misalignment, and shows how the coupling may be used as a radial bearing by examining how the radial forces vary with an increasing cone angle, $\theta$.

\section{Results and Discussion of FEM / BEM Analysis}

Figures 6 and 7 display the results of the physical testing.

The results from computational modelling show a direct correlation with the experimental results. There is a discrepancy of under $10 \%$; this is attributed to flaws in the experimental procedure and the introduction of the sample material into the air gap i.e. a change in the permeability of the gap. The modelling can therefore be seen to provide an accurate method to model these magnet configurations (Figure ). 
The axial force was calculated as a function of rotational angle $(\varnothing)$ around the z-axis. This is important as fluid forces acting on the impeller will cause lag. The results show maximal axial force for fully aligned magnets i.e. $\varnothing=0^{\circ}$. Fully misaligned magnets show zero axial force i.e. $\varnothing=45^{\circ}$ for an 8 magnet configuration ( $30^{\circ}$ for a 12 magnet configuration) (Figure). 


\section{Results and Discussion of Torsional Modelling Using FEM / BEM Analysis}

Results of the torsional modelling are displayed in Figure.

Amperes produced results showing how the torque varies between 8 and 12 magnet arrangements at different cone angles. The 12 magnet configurations produced a peak torque approximately $160 \%$ that of 8 magnet configurations. There is no difference in the torque produced when the cone angle is varied. Full parametric results showing torque at various separations are available (not shown here).

\section{Results and Discussion of Radial Force Modelling Using FEM / BEM Analysis}

The primary aim of this investigation was to show that a magnetic coupling could be designed that also acts as an axial and radial bearing. The pull-off tests provide information regarding the axial force. In order to determine the force required to disturb the coupling in a radial direction, Amperes simulated a radial pushoff test. In this test the upper component of the coupling underwent a planar translation along the y-axis. This enabled the axial and radial forces to be modelled as the upper component is pushed off-centre.

Results of radial force testing are shown in Figure 9.

For $0^{\circ}$ cone angle there is a sharp drop-off in axial force $(35 \mathrm{~N}$ to $7.5 \mathrm{~N})$ over a small displacement $(0.5$ $\mathrm{mm}$ ) whilst the radial force shows a steady increase over the range. The introduction of a cone angle produces a much more stable configuration. There is no sharp drop-off in the axial force; it remains effectively constant over the first $1 \mathrm{~mm}$ displacement. The radial force increases at a greater rate over the range. 


\section{Conclusions}

Most importantly, this investigation has shown that it is possible to design a magnetic coupling that not only acts as the drive system for a CRBP, but as a bearing system that offers both axial and radial bearing forces. It is now possible to predict the full performance characteristics for the magnet configurations tested. For example an un-backed 8 magnet configuration, separated by $5 \mathrm{~mm}$, with a $30^{\circ}$ cone angle, operating under conditions that result in a $z$-axis misalignment of $10^{\circ}$ (due to rotor lag), yields a drive system that produces an attractive force of $26 \mathrm{~N}$ with operating torque of $0.22 \mathrm{Nm}$. This is a potential configuration suggested for use in a new CRBP. The drive / bearing system may be designed by evaluating the forces that we expect to see within a CRBP (this includes forces seen by the impeller and shock forces due to external excitation of the system accounting for the mass of the impeller itself). The appropriate magnet configuration can therefore be selected to accommodate for these forces. Computational modelling enabled testing that otherwise would be difficult to perform through physical testing. It has shown that incorporation of a cone angle into the magnetic arrangement substantially increases the radial bearing force of the coupling without compromising the axial bearing force.

The work presented here is the basis for the design of a new CRBP. The next step in the design is to make the transition to an electromagnetic drive to replace the driving permanent magnets. Computational modelling may be used to fully maximise its potential for the electromagnetic design. Full modelling of the proposed electromagnetic coils is possible in order to determine the correct specifications to facilitate the conversion from permanent magnets. Once completed these electromagnets may be incorporated into the pump casing of a CRBP, and combined with the correct control system may be seen as attractive solution for the drive / bearing of the rotor. 


\section{Acknowledgements}

Our thanks go out to the many staff at The University of Nottingham whose continued help allows for the

furtherance of this research. This project is sponsored by the University of Nottingham and Engineering and Physical Sciences Research Council (EPSRC) through a Doctoral Training Award. 


\section{References}

1. Furlani EP. Permanent Magnet and Electromechanical Devices, $1^{\text {st }}$ edition. Academic Press, 2001.

2. Delamare J, Yonnet JP, Rullière E. A Compact Magnetic Suspension with Only One Axis Control. IEEE Transactions on Magnetics 1994; 30(6): 4746-4748.

3. Yonnet JP. Permanent Magnet Bearings and Couplings. IEEE Transactions on Magnetics $1981 ; 17(1)$ : 1169-1173.

4. Nosé Y, Yoshikawa M, Murabayashi S, Takano T. Development of Rotary Blood Pump Technology:

Past, Present, and Future. Artificial Organs 2000; 24(6): 412-420. 


\section{Tables}

Table 1: Number of magnets tested at different cone angles under different backing conditions. 


\section{Figure legends}

Figure 1: Possible configurations of ring magnets and magnetic flux density vectors to achieve magnetic suspension.

Figure 2: a) Ring magnet bearing. b) Rings substituted for disc magnets to achieve the same bearing configuration.

Figure 3: a) Angular alignment around the z-axis, $\varnothing$. b) Cone angle of magnets $\theta$. Figure 4: Magnetic pull-off tests shown at $5 \mathrm{~mm}$ separation. a) 12 magnets at $0^{\circ}$ cone angle $\theta$. b) 12 magnets at $30^{\circ}$ cone angle. Note rotational displacement around the z-axis (i.e. in $\emptyset$ ) is zero.

Figure 6: Comparison between experimental and computational results show a direct correlation with an increasing difference at smaller separations.

Figure 7: Axial force at $\mathrm{z}$-axis misalignment, $\Phi$. As the coupling is forced into misalignment the axial force decreases.

Figure 8: Amperes results for torque about the z-axis. 12 magnet configuration shows a larger torque than 8 magnets. No difference in torque with the introduction of a cone angle, $\theta$.

Figure 9: Amperes translation along the y-axis. Introduction of cone angle increases the radial force required to disturb the coupling. 

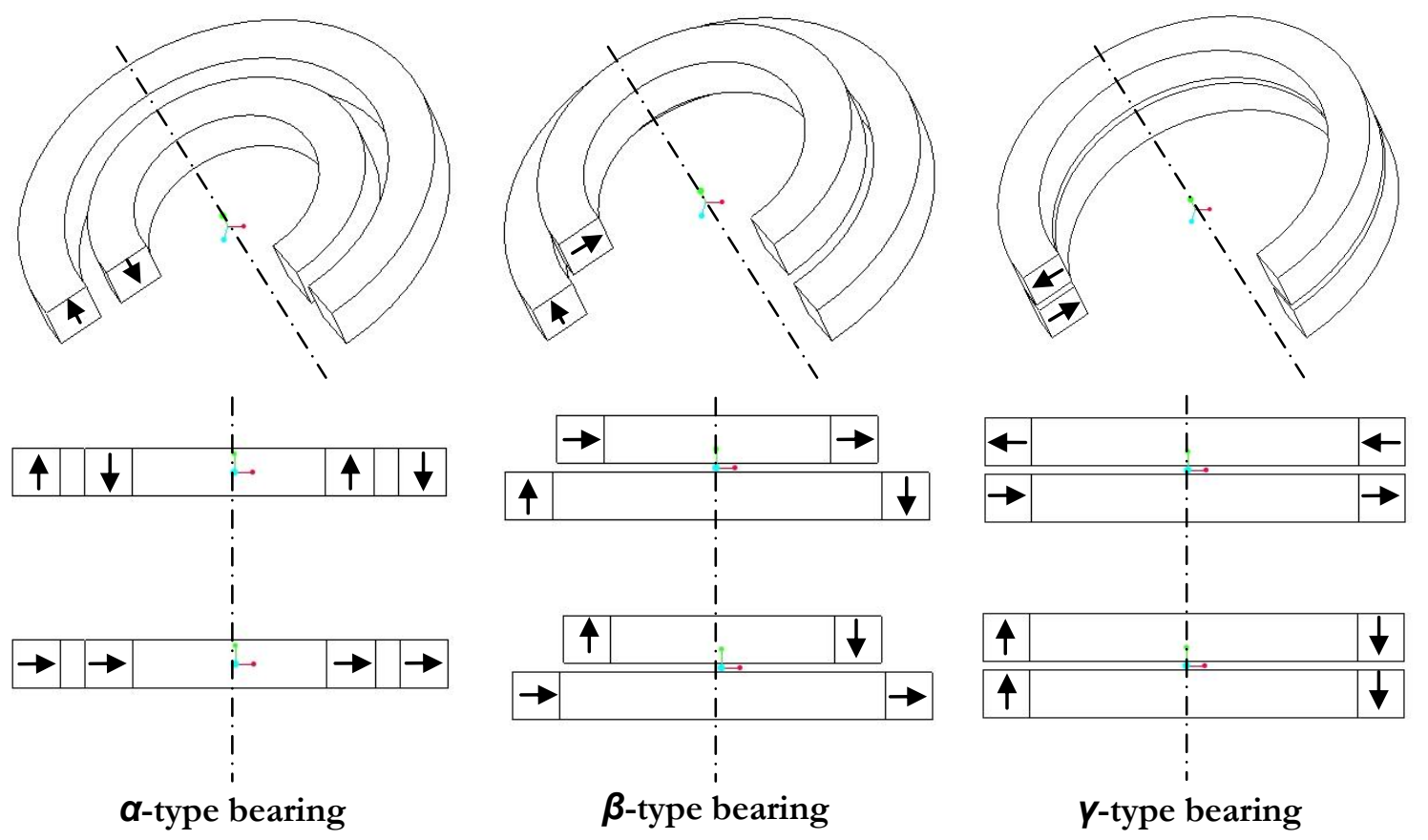

$\uparrow \quad$ Magnetic Flux Density Vector, $B_{r}$

Figure 1: Possible configurations of ring magnets and magnetic flux density vectors to achieve magnetic suspension 

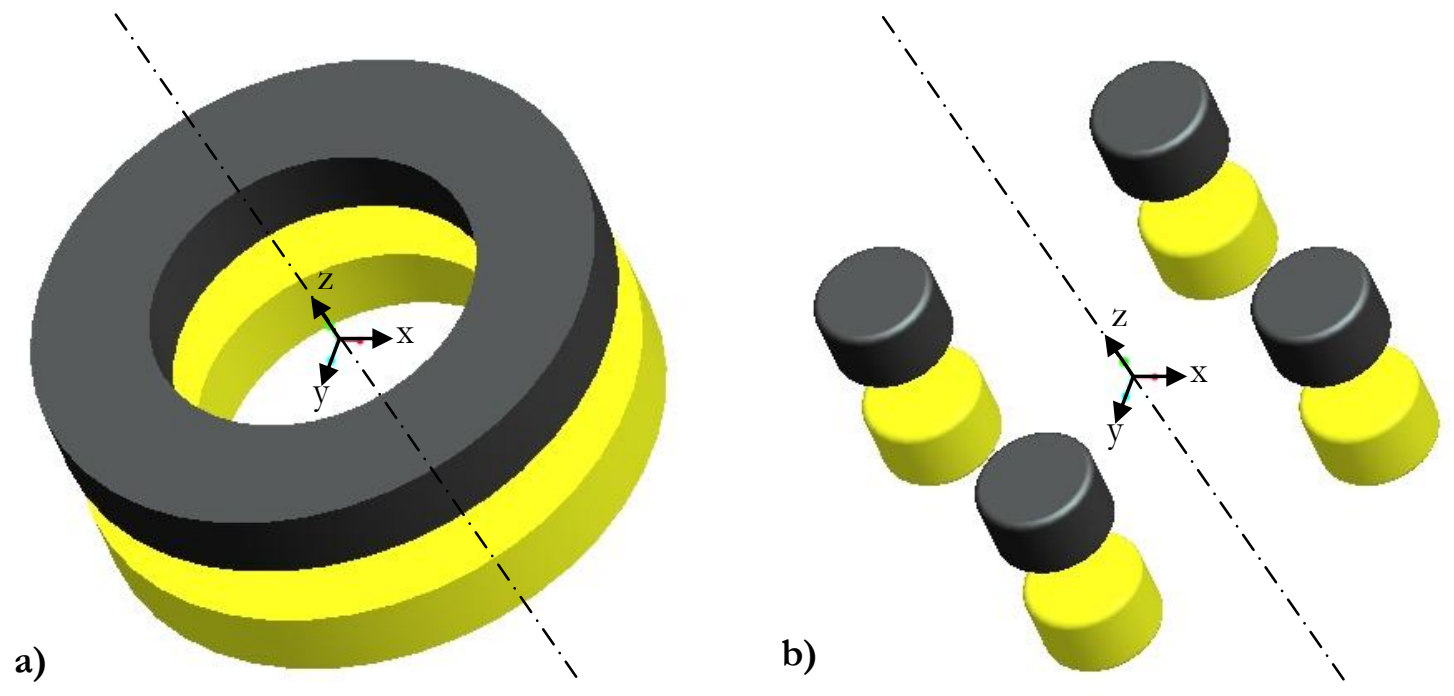

Figure 2: a) Ring magnet bearing. b) Rings substituted for disc magnets to achieve the same bearing configuration. 

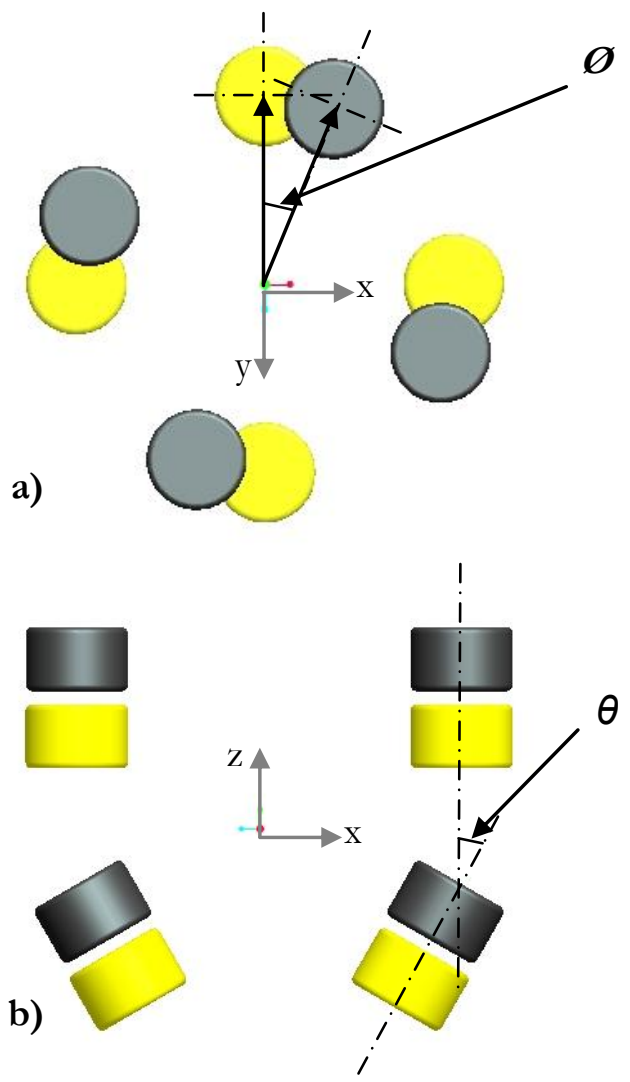

Figure 3: a) Angular alignment around the z-axis, $\varnothing$. b) Cone angle of magnets $\theta$. 


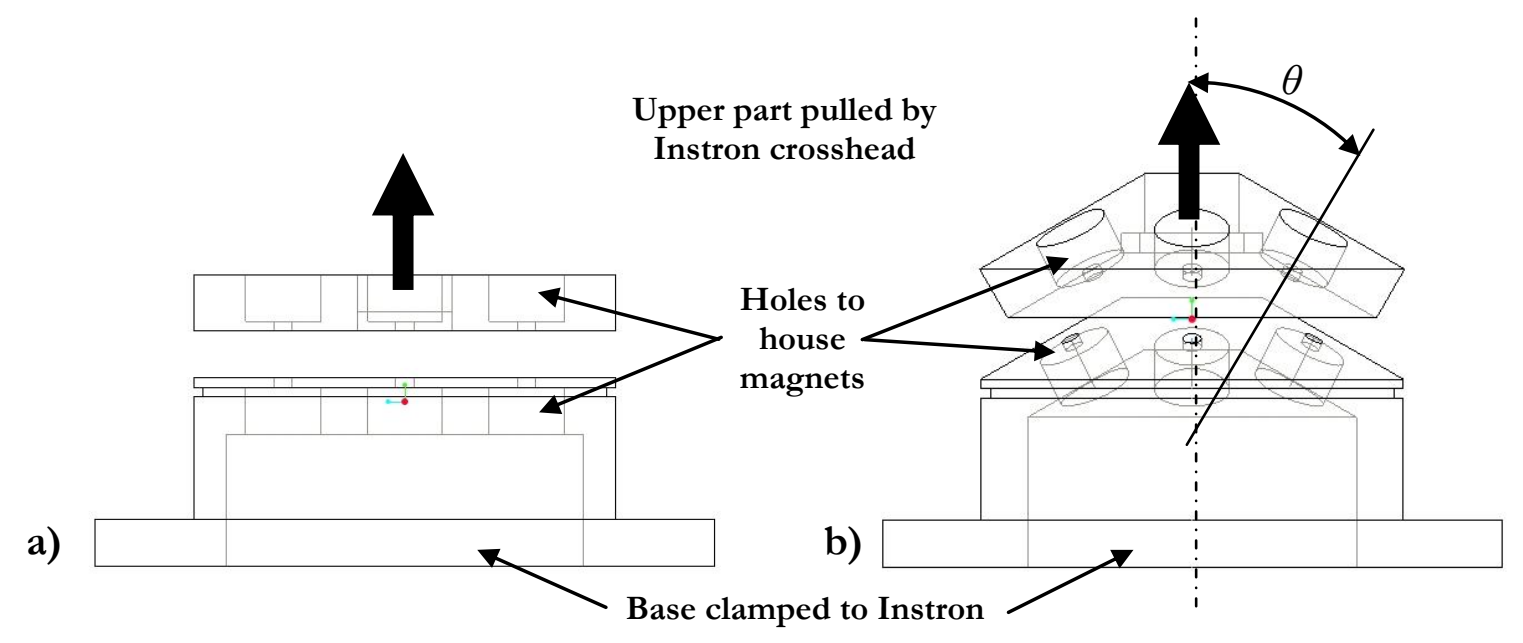

Figure 4: Magnetic pull-off tests shown at $5 \mathrm{~mm}$ separation. a) 12 magnets at $0^{\circ}$ cone angle $\theta$. b) 12 magnets at $30^{\circ}$ cone angle. Note rotational displacement around the z-axis (i.e. in $\varnothing)$ is zero 

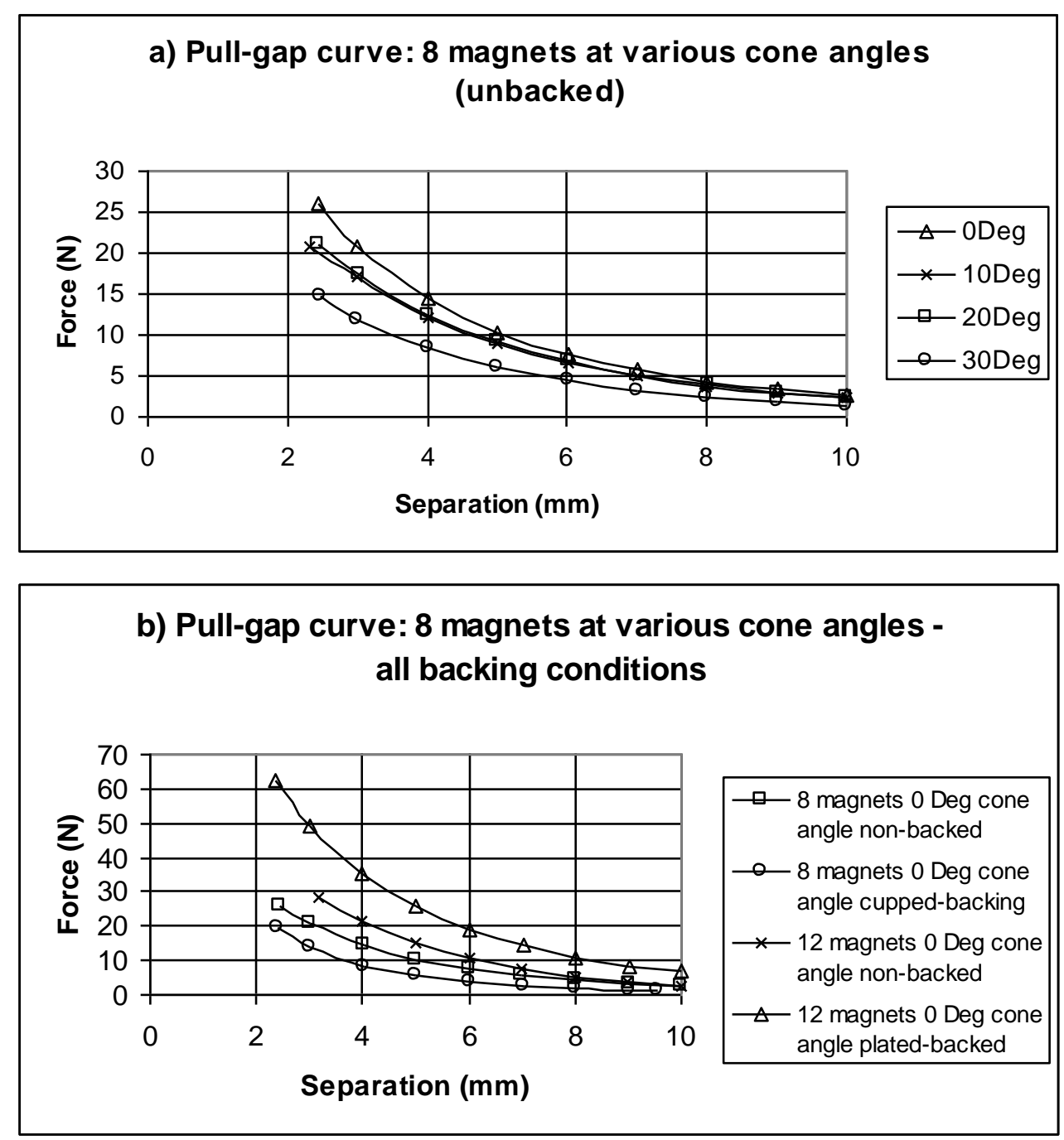

Figure 5: Pull-gap curves. a) 8 magnets at various cone angles.

b) 8 and 12 magnets under different backing conditions. 


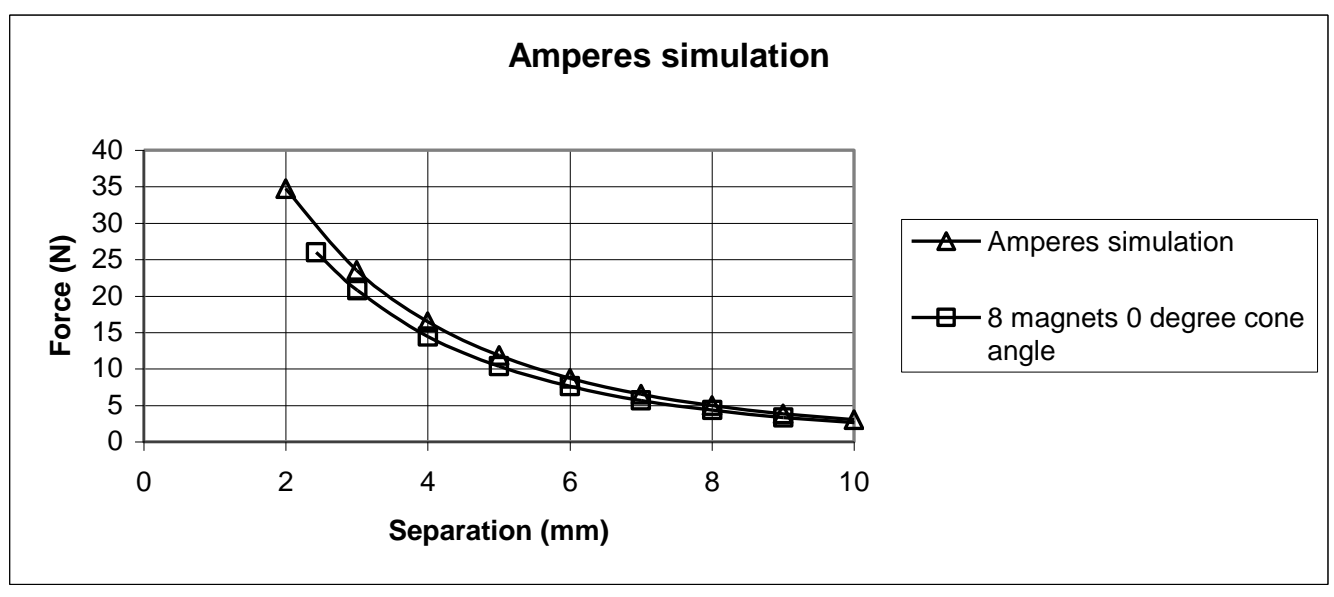

Figure 6: Comparison between experimental and computational results show a direct correlation with an increasing difference at smaller separations. 


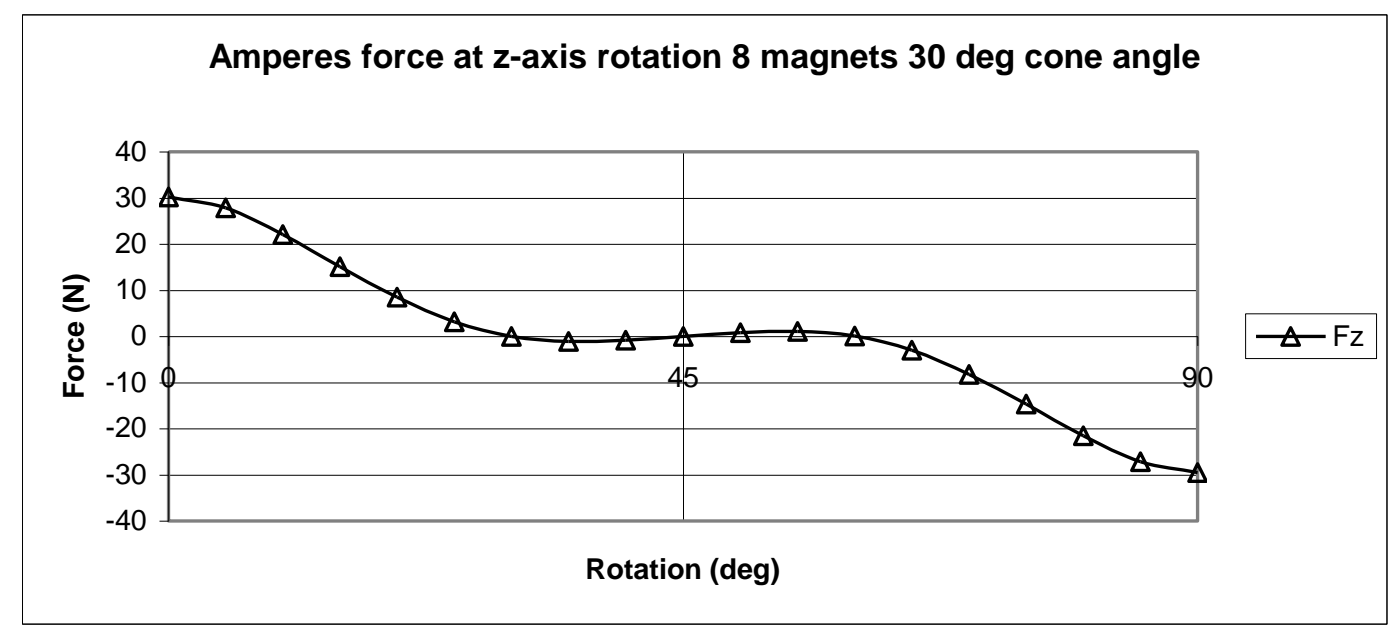

Figure 7: Axial force at z-axis misalignment, $\Phi$. As the coupling is forced into misalignment the axial force decreases. 


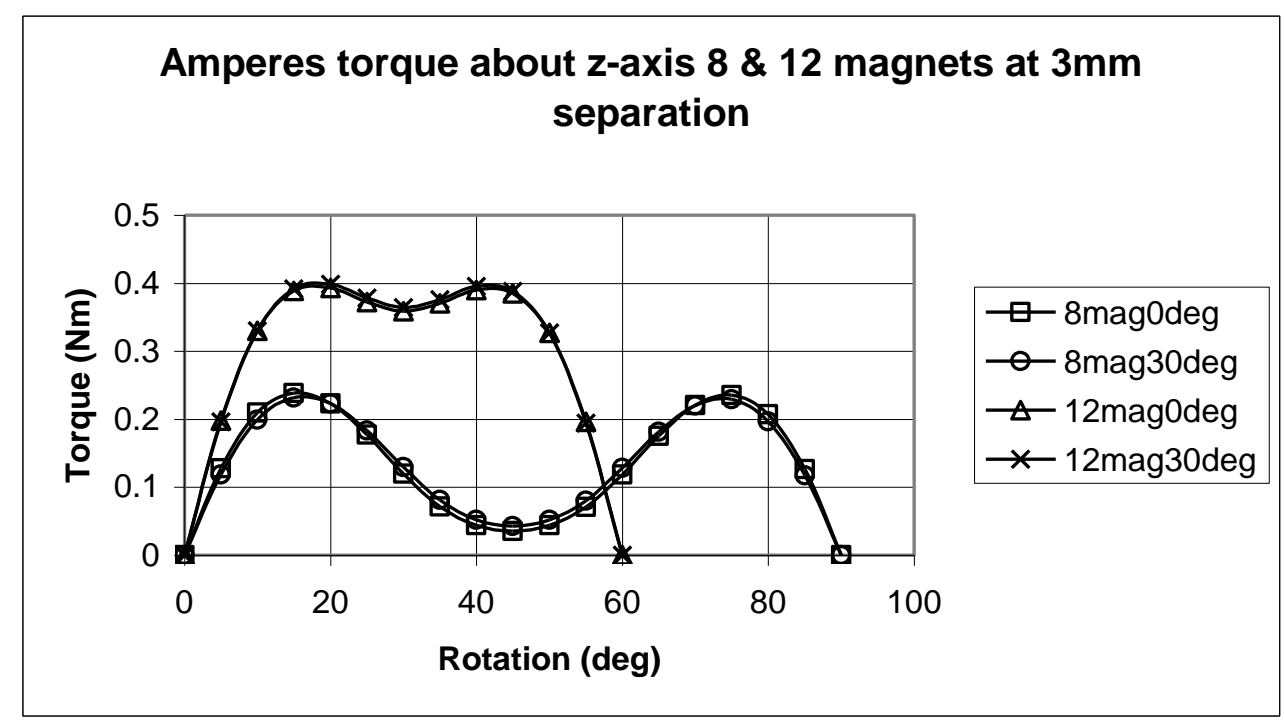

Figure 8: Amperes results for torque about the z-axis. 12

magnet configuration shows a larger torque than 8 magnets.

No difference in torque with the introduction of a cone angle, $\boldsymbol{\theta}$. 


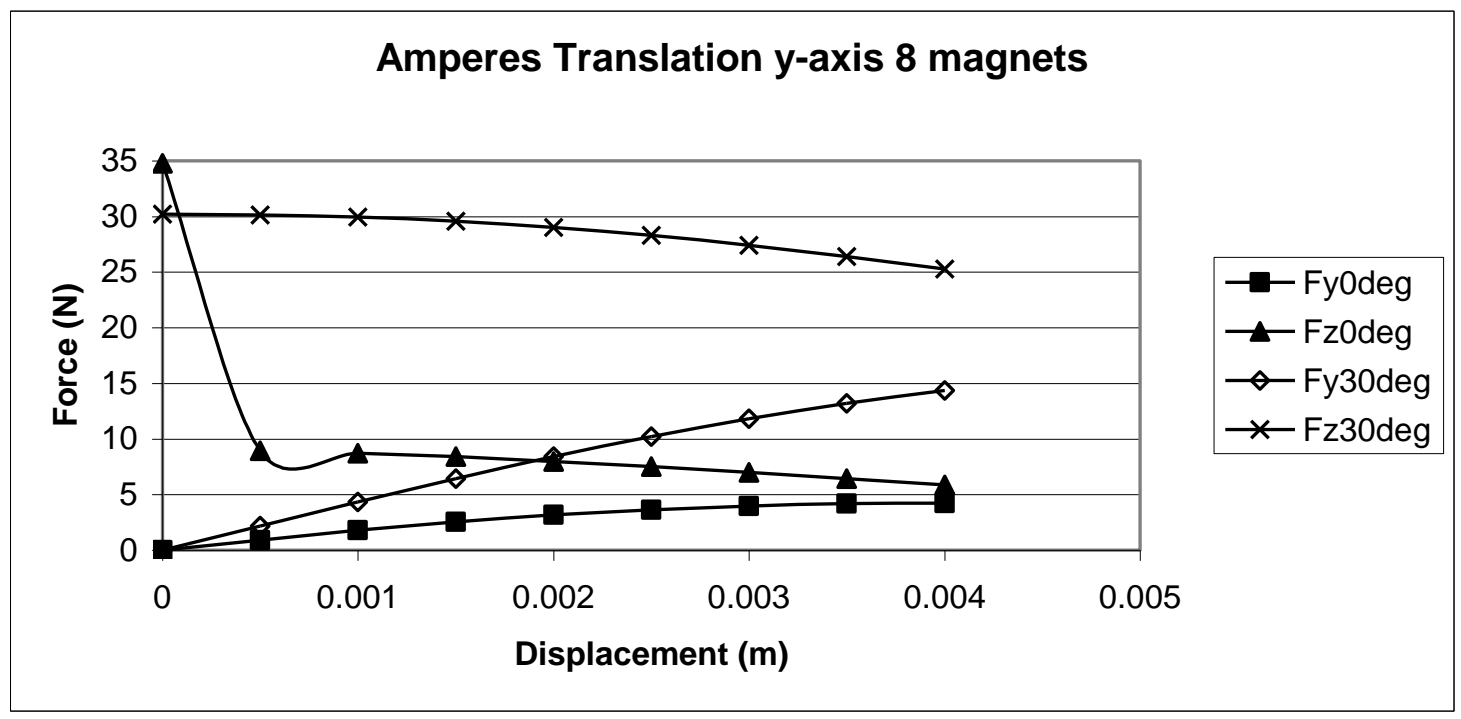

Figure 9: Amperes translation along the y-axis. Introduction of cone angle increases the radial force required to disturb the coupling. 
Table 1: Number of magnets tested at different cone angles under different backing conditions

\begin{tabular}{|c|c|c|c|c|}
\cline { 2 - 5 } \multicolumn{1}{c|}{} & \multicolumn{4}{c|}{ Angle $\theta$ (Degrees) } \\
\hline $\begin{array}{c}\text { Backing } \\
\text { Type }\end{array}$ & 0 & 10 & 20 & 30 \\
\cline { 1 - 1 } Non-backed & 8,12 & 8,12 & 8,12 & 8,12 \\
\cline { 1 - 1 } $\begin{array}{c}\text { Cupped } \\
\text { backing }\end{array}$ & 8 & 8 & 8 & 8 \\
\cline { 1 - 1 } $\begin{array}{c}\text { Steel plate } \\
\text { back iron }\end{array}$ & 12 & N/A & N/A & N/A \\
\hline
\end{tabular}

